\title{
Performance Analysis of the MRC M2M System
}

\author{
Lingwei $\mathrm{Xu}^{1}$, Hao Zhang ${ }^{1,2}$, Tingting $\mathrm{Lu}^{1}$, Xing Liu ${ }^{1}$, and T. Aaron Gulliver ${ }^{2}$ \\ ${ }^{1}$ College of Information Science and Engineering, Ocean University of China, \\ Qingdao 266100, China \\ ${ }^{2}$ Department of Electrical and Computer Engineering, University of Victoria, \\ Victoria V8W 2Y2, Canada \\ gaomilaojia2009@163.com; zhanghao@ouc.edu.cn; lvtingting33@163.com, \\ liuxing3901@163.com,agullive@ece.uvic.ca
}

\begin{abstract}
The average symbol error probability (ASEP) of a mobile-to-mobile (M2M) system employing maximal ratio combining (MRC) over N-Nakagami fading channels is investigated in this paper. The characteristic function $(C F)$ of the signal-to-noise ratio (SNR) at the MRC output is used to derive exact ASEP expressions for several q-ary modulation schemes, including phase shift keying (PSK) modulation, quadrature amplitude modulation (QAM), and pulse amplitude modulation (PAM). Then the ASEP performance under different channel conditions is evaluated through numerical simulation to verify the analysis. These results show that the performance of the MRC M2M system is improved as the number of diversity branches or the fading coefficient is increased. Conversely, performance is degraded as the number of cascaded components is increased.
\end{abstract}

Keywords: $M 2 M$ communications, maximal ratio combining, N-Nakagami fading channels, average symbol error probability, characteristic function

\section{Introduction}

Mobile-to-mobile (M2M) communication has attracted wide research interest in recent years. It is widely employed in wireless communication systems, such as mobile ad-hoc and vehicle-to-vehicle networks [1]. When both the transmitter and receiver are moving, the channel can be characterized by a double-Rayleigh fading model [2]. Extending this model to the more realistic Nakagami fading, a double-Nakagami fading model has also been considered [3]. A generalization of this model, the N-Nakagami distribution, which is the product of $\mathrm{N}$ Nakagami random variables which are statistically independent but not necessarily identically distributed, was introduced and analyzed in [4].

To improve the spectral efficiency and reliability of M2M communication systems, multiple-input multiple-output (MIMO) technology can be employed to improve the capacity or spatial diversity [5]. It is known that spatial diversity techniques such as maximal ratio combining (MRC), equal gain combining (EGC), and selection combining (SC) can mitigate the detrimental effects of fading. MRC is the optimal diversity combining technique which maximizes the signal-to-noise (SNR) [6].

M2M systems employing diversity techniques have been investigated in the literature. The performance of the MRC M2M systems over non-identical double-Rayleigh fading channels was derived using the moment generating function (MGF) approach in [7]. The average bit error probability (BEP) and the outage probability of the MRC M2M systems over double-Nakagami channels was derived using the MGF approach in [8]. This approach was also employed to study the effect of MRC and SC over N-Weibull channels [9]. In [10], the pairwise error probability (PEP) for a cooperative inter-vehicular communication (IVC) system over double-Nakagami fading channels was derived. The 
SER for an M2M system with decode-and-forward (DF) relaying over double-Nakagami fading channels was investigated in [11] using the MGF approach.

To date, the analysis of M2M communication systems has concentrated on the performance of specific diversity combining techniques using the MGF method and the probability density function (PDF) of the SNR in cascaded channels. However, it is difficult to obtain a closed form expression for the PDF and cumulative density function (CDF) of the SNR at the MRC output over N-Nakagami fading channels. In this case, the characteristic function (CF) method can be used to overcome the limitations of these methods [12]. Thus, in this paper the CF method is used to derive exact average symbol error probability (ASEP) expressions for an M2M communication system employing MRC over N-Nakagami fading channels.

The rest of the paper is organized as follows. The M2M communication system employing MRC is presented in Section 2. Section 3 provides exact ASEP expressions for several q-ary modulation schemes, including PSK, QAM, and PAM. Section 4 presents simulation results to verify and illustrate the ASEP performance. Finally, some concluding remarks are given in Section 5.

\section{The System Model}

Consider a product of $N$ independent random variables

$$
Z=\prod_{i=1}^{N} a_{i}
$$

where $N$ is the number of cascaded components, and $a_{i}$ is a Nakagami distributed random variable with $\mathrm{PDF}$

$$
f(a)=\frac{2 m^{m}}{\Omega^{m} \Gamma(m)} a^{2 m-1} \exp \left(-\frac{m}{\Omega} a^{2}\right)
$$

where $\Gamma(\cdot)$ is the Gamma function, $m$ is the Nakagami fading coefficient, and $\Omega$ is a scaling factor.

The PDF of $Z$ is given by [4]

$$
f_{Z}(z)=\frac{2}{z \prod_{i=1}^{N} \Gamma\left(m_{i}\right)} G_{0, N}^{N, 0}\left\lfloor\left. z^{2} \prod_{i=1}^{N} \frac{m_{i}}{\Omega_{i}}\right|_{m_{1}, \ldots, m_{N}} ^{-}\right\rfloor
$$

where G[·] is Meijer's G-function [4].

Consider an M2M communication system employing MRC over N-Nakagami fading channels in the presence of additive white Gaussian noise (AWGN). We assume there are $L$ independent diversity branches. The instantaneous SNR for the $l$ th branch is given by

$$
r_{l}=\left|Z_{l}\right|^{2} \frac{E_{s}}{N_{0}}, l=1, \ldots, L
$$

where $E s$ is the average energy of a transmitted symbol and $N_{0}$ is the single-sided AWGN power spectral density. The corresponding average SNR is

$$
\overline{r_{l}}=E\left(\left|Z_{l}\right|^{2}\right) \frac{E_{s}}{N_{0}}, l=1, \cdots, L
$$

where $E()$ denotes expectation.

The CDF of $r_{l}$ can be derived as [4] 


$$
F_{r_{i}}(r)=\frac{1}{\prod_{i=1}^{N} \Gamma\left(m_{i}\right)} G_{1, N+1}^{N, 1}\left\lceil\left.\stackrel{r}{=} \prod_{r_{l}}^{N} m_{i=1}^{N} m_{i}\right|_{m_{1}, \ldots, m_{N}, 0} ^{1}\right\rfloor
$$

By taking the first derivative of (6) with respect to $r$, the corresponding PDF can be obtained as

$$
f_{r_{l}}(r)=\frac{1}{r \prod_{i=1}^{N} \Gamma\left(m_{i}\right)} G_{0, N}^{N, 0}\left\lceil\left.\frac{r}{=} \prod_{i=1}^{N} m_{i}\right|_{m_{1}, \ldots, m_{N}} ^{-}\right\rfloor
$$

The SNR at the MRC output is then [13]

$$
r_{M R C}=\sum_{l=1}^{L} r_{l}=\sum_{l=1}^{L}\left|Z_{l}\right|^{2} \frac{E_{s}}{N_{0}}
$$

An $L$-fold convolution of expressions of the form in (7) can be used to obtain the PDF of $r_{\mathrm{MRC}}$. Since it is very difficult to perform this convolution, the CF method is used here to obtain the average SEP performance.

\section{Average Symbol Error Probability}

The ASEP using the CF method is given by [12]

$$
P=\frac{1}{2 \pi} \int_{-\infty}^{\infty} G_{x}(w) \psi_{x}(w, L) d w
$$

where

$$
G_{x}(w)=\int_{0}^{\infty} P_{q}(x) \exp (-j w x) d x
$$

and

$$
\psi_{x}(w, L)=\int_{-\infty}^{\infty} f(x, L) \exp (j w x) d x
$$

$P q(x)$ denotes the symbol error probability for $q$-ary modulation in an AWGN channel, $\Psi x(w, L)$ denotes the CF of the random variable $x$ at the combiner output, and $f(x, L)$ denotes the PDF of $x$ at the combiner output.

For numerical integration, the substitution $w=\tan (\theta)$ in (9) can be used to obtain [12]

$$
P=\frac{1}{\pi} \int_{0}^{\pi / 2} \xi\left\{G_{x}(\tan (\theta)) \psi_{x}(\tan (\theta), L)\right\} \sec ^{2}(\theta) d \theta
$$

where $\xi$ denotes the real part of the argument.

From the CF of $r_{\mathrm{MRC}}$, it is straightforward to show that [12]

$$
\psi^{(M R C)}(w, L)=\prod_{l=1}^{L} \psi_{l}(w)
$$

where $\Psi_{l}(w)$ denotes the CF of the SNR of the lth diversity branch. We consider the case where the branches are independent and identical, each with a PDF and CDF given by (6) and (7), respectively. The average SNR for all branches can be expressed as

Substituting (7) into (11) gives

$$
\overline{r_{l}}=\bar{r}
$$




$$
\psi_{l}(w)=\frac{1}{\prod_{i=1}^{N} \Gamma\left(m_{i}\right)} \int_{-\infty}^{\infty} \frac{\exp (j w r)}{r} G_{0, N}^{N, 0}\left\lceil\left.\stackrel{r}{=} \prod_{i=1}^{N} m_{i}\right|_{m_{1}, \ldots, m_{N}} ^{-}\right\rceil d r
$$

To evaluate the integral in (15), the following integral function can be employed [14]

$$
\begin{aligned}
& \exp (-x)=G_{0,1}^{1,0}\left[\left.x\right|_{0} ^{-}\right] \\
& \int_{0}^{\infty} x^{a-1} G_{u, v}^{s, t}\left[\sigma x \mid \begin{array}{l}
c_{1}, \cdots, c_{u} \\
a_{1}, \cdots, d_{v}
\end{array}\right] G_{p, q}^{m, n}\left[w x^{\frac{l}{k}} \mid \begin{array}{l}
a_{1}, \cdots, a_{p} \\
b_{1}, \cdots, b_{q}
\end{array}\right] d x \\
& =\frac{k^{\phi} l^{\theta+a(v-u)-1} \sigma^{-a}}{(2 \pi)^{b^{(}(l-1)+c^{*}(k-1)}} G_{k p+l v, k q+l u}^{k m+l, k n+l}\left\lceil\frac{w^{k} k^{k(p-q)}}{\sigma^{l} l^{l(u-v)}} \mid \begin{array}{l}
\Delta\left(k, a_{1}\right), \cdots, \Delta\left(k, a_{n}\right), \\
\Delta\left(k, b_{1}\right), \cdots, \Delta\left(k, b_{m}\right),
\end{array}\right. \\
& \left.\Delta\left(l, 1-a-d_{1}\right), \cdots, \Delta\left(l, 1-a-d_{v}\right), \Delta\left(k, a_{n+1}\right), \cdots, \Delta\left(k, a_{p}\right)\right\rceil \\
& \left.\Delta\left(l, 1-a-c_{1}\right), \cdots, \Delta\left(l, 1-a-c_{u}\right), \Delta\left(k, b_{m+1}\right), \cdots \Delta\left(k, b_{q}\right)\right\rfloor
\end{aligned}
$$

where $0 \leq m \leq q, 0 \leq n \leq p, 0 \leq s \leq v, 0 \leq t \leq u$, and they are integers. $a_{i}, b_{i}, c_{i}$ and $d_{i}$ are arbitrary real values, and

$$
\begin{aligned}
& b^{*}=s+t-\frac{u+v}{2} \\
& c^{*}=m+n-\frac{p+q}{2} \\
& \phi=\sum_{i=1}^{p} b_{i}-\sum_{i=1}^{q} b_{i}+\frac{p-q}{2}+1 \\
& \theta=\sum_{i=1}^{v} d_{i}-\sum_{i=1}^{u} c_{i}+\frac{u-v}{2}+1 \\
& \Delta\left(k, a_{n}\right)=\frac{a_{n}}{k}, \frac{a_{n}+1}{k}, \cdots, \frac{a_{n}+k-1}{k}
\end{aligned}
$$

Equation (15) can be written as

$$
\begin{aligned}
& \psi_{i}(w)=\frac{1}{\prod_{i=1}^{N} \Gamma\left(m_{i}\right)} \int_{-\infty}^{\infty} \frac{\exp (j w r)}{r} G_{0, N}^{N, 0}\left[\left.\begin{array}{r}
r \\
r
\end{array} \prod_{i=1}^{N} m_{i}\right|_{m_{1}, \ldots, m_{N}} ^{-}\right\rceil d r \\
& =\frac{1}{\prod_{i=1}^{N} \Gamma\left(m_{i}\right)} \int_{-\infty}^{\infty} r^{-1} G_{0,1}^{1,0}\left[-\left.j w r\right|_{0} ^{-}\right] G_{0, N}^{N, 0}\left\lceil\left.\frac{r}{r} \prod_{i=1}^{N} m_{i}\right|_{m_{1}, \ldots, m_{N}} ^{-}\right\rceil d r \\
& =\frac{1}{\prod_{i=1}^{N} \Gamma\left(m_{i}\right)} G_{1, N}^{N, 1}\left\lceil\left.\frac{j}{w r} \prod_{i=1}^{N} m_{i}\right|_{m_{1}, \ldots, m_{N}} ^{1}\right\rceil
\end{aligned}
$$

and

$$
\begin{aligned}
& \psi^{(M R C)}(w, L)=\left(\psi_{l}(w)\right)^{L}
\end{aligned}
$$

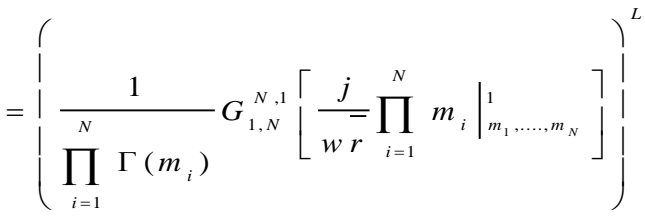

\section{1. $q$-PAM}

The symbol error probability (SEP) of $q$-ary pulse amplitude modulation (PAM) in an 
AWGN channel is given by [15]

$$
P_{q}(r)=2\left(1-\frac{1}{q}\right) Q\left(\sqrt{\frac{6 r}{q^{2}-1}}\right)
$$

Substituting (21) into (10), we obtain

$$
\begin{aligned}
& G_{x}(w)=2\left(1-\frac{1}{q}\right) \int_{0}^{\infty} Q\left(\sqrt{\frac{6 r}{q^{2}-1}}\right) \exp (-j w r) d r \\
& =\left(1-\frac{1}{q}\right) \int_{0}^{\infty} \operatorname{erfc}\left(\sqrt{\frac{3 r}{q^{2}-1}}\right) \exp (-j w r) d r \\
& =\frac{(q-1)}{j w q}\left(1-\sqrt{\frac{3}{3+j w\left(q^{2}-1\right)}}\right)
\end{aligned}
$$

Next, substituting (20) and (22) into (12) gives the average SEP of $q$-ary PAM over NNakagami fading channels as

$$
\begin{aligned}
& P_{P A M}= \frac{1-\frac{1}{q}}{\pi\left(\prod_{i=1}^{N} \Gamma\left(m_{i}\right)\right)^{L} \times} \\
& \int_{0}^{\pi / 2} \xi\left\{\begin{array}{l}
\frac{1}{j \tan (\theta)}\left(1-\sqrt{\frac{3}{3+j\left(q^{2}-1\right) \tan (\theta)}}\right) \times \mid \\
\left\{\left(G_{1, N}^{N, 1}\left\lceil\left.\frac{j}{\tan (\theta) r} \prod_{i=1}^{N} m_{i}\right|_{m_{1}, \ldots, m_{N}} ^{1}\right]\right)^{2}\right\}
\end{array}\right\} \sec ^{2}(\theta) d \theta
\end{aligned}
$$

\section{2. $q$-PSK}

The SEP of $q$-ary phase shift keying (PSK) modulation in an AWGN channel is given by [15]

$$
P_{q}(r)=2 Q\left(\sqrt{2 \sin ^{2} \frac{\pi}{q} r}\right)-\frac{1}{\pi} \int_{\frac{\pi}{2}-\frac{\pi}{q}}^{\frac{\pi}{2}} e^{-r \frac{\sin ^{2} \pi / q}{\cos ^{2} \theta}} d \theta
$$

For a sufficiently large SNR and large values of $q$, the SEP of $q$-ary PSK in an AWGN channel can be approximated as

$$
P_{q}(r) \approx 2 Q\left(\sqrt{2 \sin ^{2} \frac{\pi}{q} r}\right)
$$

Substituting (25) into (10) gives

$$
\begin{aligned}
& G_{x}(w)=2 \int_{0}^{\infty} Q\left(\sqrt{2 \sin ^{2} \frac{\pi}{q} r}\right) \exp (-j w r) d r \\
& =\int_{0}^{\infty} \operatorname{erfc}\left(\sqrt{\sin ^{2} \frac{\pi}{q} r}\right) \exp (-j w r) d r \\
& \left.=\frac{1}{j w} \mid 1-\sqrt{\frac{\sin ^{2} \frac{\pi}{q}}{\sin ^{2} \frac{\pi}{q}+j w}}\right)
\end{aligned}
$$


Now, substituting (20) and (26) into (12), the average SEP of $q$-ary PSK over NNakagami fading channels can be approximated as

$$
\begin{aligned}
& P_{P S K}(r) \approx \frac{1}{\pi\left(\prod_{i=1}^{N} \Gamma\left(m_{i}\right)\right)^{L}} \times
\end{aligned}
$$

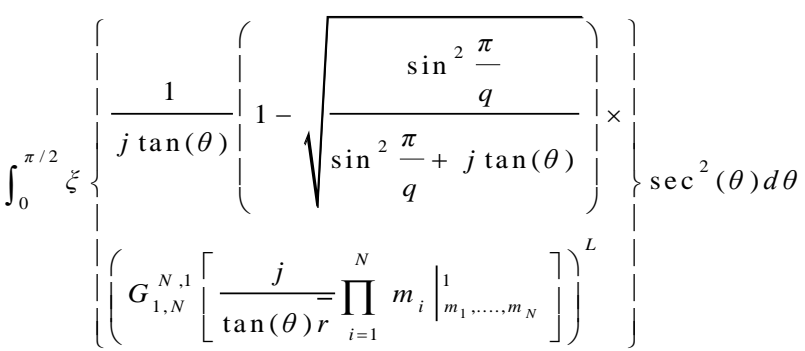

\section{3. $q$-QAM}

Rectangular quadrature amplitude modulation (QAM) signal constellations are frequently employed because they provide high bandwidth efficiency with good performance. QAM is equivalent to two PAM signals on quadrature carriers. For $q$-ary, $q=2 \mathrm{k}$ ( $k$ even), rectangular QAM, the SEP in an AWGN channel is given by [15]

$$
\begin{aligned}
& P_{q}(r)=1-\left(1-P_{P A M}, \sqrt{q}\right)^{2} \\
& =2 P_{P A M} \sqrt{q}-\left(P_{P A M} \sqrt{q}\right)^{2}
\end{aligned}
$$

where

$$
P_{P A M, \sqrt{q}}=2\left(1-\frac{1}{\sqrt{q}}\right) Q\left(\sqrt{\frac{3 r}{q-1}}\right)
$$

Substituting (28) into (10) gives

$$
\begin{aligned}
& G_{x}(w)=2\left(1-\frac{1}{\sqrt{q}}\right) \int_{0}^{\infty} \operatorname{erfc}\left(\sqrt{\frac{1.5 r}{q-1}}\right) \exp (-j w r) d r \\
& -\left(1-\frac{1}{\sqrt{q}}\right)^{2} \int_{0}^{\infty} \operatorname{erfc}^{2}\left(\sqrt{\frac{1.5 r}{q-1}}\right) \exp (-j w r) d r \\
& =\frac{2(\sqrt{q}-1)}{j w \sqrt{q}}\left(1-\sqrt{\frac{1.5}{1.5+j w(q-1)}}\right)- \\
& \frac{(\sqrt{q}-1)^{2}}{j w q}\left(1-\frac{4 \tan ^{-1}(1+j w(q-1) / 1.5)}{\pi(1+j w(q-1) / 1.5)}\right)
\end{aligned}
$$

Next, substituting (20) and (30) into (12), the average SEP of $q$-ary QAM over NNakagami fading channels is 


$$
\begin{aligned}
& P_{Q A M}=\frac{2\left(1-\frac{1}{\sqrt{q}}\right)}{\pi\left(\prod_{i=1}^{N} \Gamma\left(m_{i}\right)\right)^{L}} \times
\end{aligned}
$$

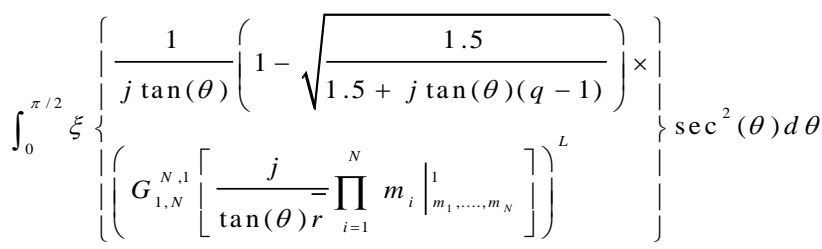

$$
\begin{aligned}
& -\frac{\left(1-\frac{1}{\sqrt{q}}\right)^{2}}{\pi\left(\prod_{i=1}^{N} \Gamma\left(m_{i}\right)\right)^{L} \int_{0}^{\pi / 2} \sec ^{2}(\theta) \times} \\
& \xi\left\{\begin{array}{ll}
\left|\frac{1}{j \tan (\theta)}\left(1-\frac{4 \tan ^{-1}(1+j \tan (\theta)(q-1) / 1.5)}{\pi(1+j \tan (\theta)(q-1) / 1.5)}\right) \times\right| & \mid \\
\mid\left\{G_{1, N}^{N, 1}\left\lfloor\left.\frac{j}{\tan (\theta) r} \prod_{i=1}^{N} m_{i}\right|_{m_{1}, \ldots, m_{N}} ^{1}\right\rceil\right) & \mid
\end{array}\right\}
\end{aligned}
$$

\section{Numerical Results}

In this section, numerical results are presented to illustrate and verify the ASEP results obtained in the previous sections.

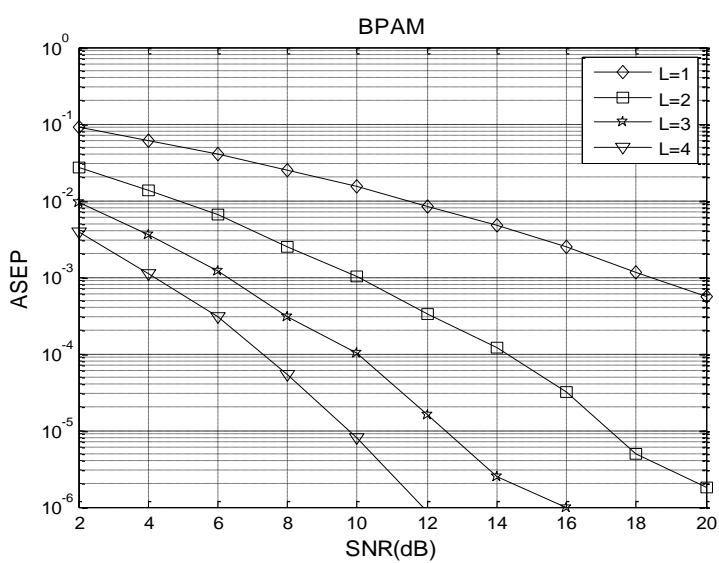

Figure 1. The Impact of Diversity on the Average SEP

Figure 1 presents the impact of diversity on the average SEP of the M2M communication system employing MRC over N-Nakagami fading channels with binary PAM. The number of cascaded components is $N=2$, and the fading coefficient is $m=2$. The number of diversity branches is $L=1,2,3,4$. These results show that the SEP performance is improved as the diversity branches $L$ increases, as expected. For example, at $S N R=10$ $\mathrm{dB}$, when $L=1$ the average SEP is $1.5 \times 10^{-2}$, with $L=2$ the average SEP is $1 \times 10^{-3}$, with $L=3$ the average SEP is $1 \times 10^{-4}$, and with $L=4$ the average SEP is $8 \times 10^{-6}$, which is significantly lower. An increase in the SNR decreases the average SEP over N-Nakagami fading channels, but the change is approximately linear. 


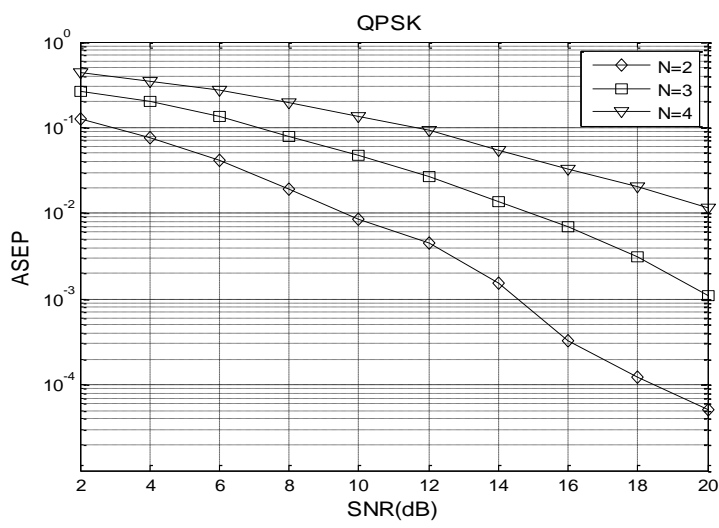

\section{Figure 2. The Impact of the Number of Cascaded Components on the Average SEP}

Figure 2 presents the impact of the number of cascaded components $N$ on the average SEP of the M2M communication system employing MRC over N-Nakagami fading channels with QPSK modulation. The number of cascaded components is $N=2,3,4$. The number of diversity branches is $L=2$, and the fading coefficient is $m=2$. These results show that the average SEP is degraded as the number of cascaded components $N$ is increased. This indicates worsening channel conditions as $N$ increases. For example, at $\mathrm{SNR}=12 \mathrm{~dB}$, when $N=2$ the average SEP is $4 \times 10^{-3}$, when $N=3$, the average SEP is $3 \times 10^{-2}$, and when $N=4$ the ASEP is $9 \times 10^{-2}$. As observed previously, an increase in the SNR results in a reduction of the ASEP over N-Nakagami fading channels.

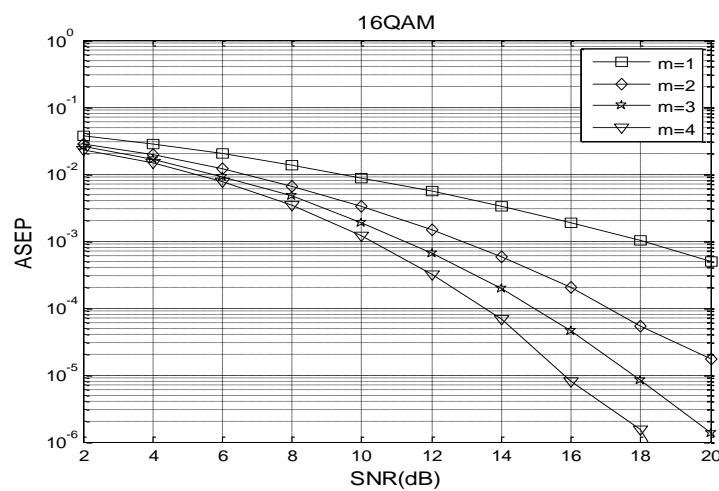

\section{Figure 3. The Impact of the Fading Coefficient $m$ on the Average SEP}

Figure 3 presents the impact of the fading coefficient $m$ on the average SEP of the M2M communication system employing MRC over N-Nakagami fading channels with 16 QAM. The fading coefficient is $m=1,2,3,4$. The number of diversity branches is $L=2$, and the number of cascaded components is $N=2$. These results show that the average SEP performance improves as the fading coefficient $m$ increases. For example, if $S N R=14 \mathrm{~dB}$, with $m=1$ the average SEP is $3 \times 10^{-3}$, with $m=2$ the average SEP is $5 \times 10^{-4}$, with $m=3$ the average SEP is $2 \times 10^{-4}$, and with $m=4$ the average SEP is $6 \times 10^{-5}$, which is a significant improvement over $m=1$. Increasing the SNR again reduces the average SEP.

\section{Conclusion}

The ASEP of a M2M communication system employing maximal ratio combining (MRC) over N-Nakagami fading channels was investigated. Exact ASEP expressions were derived. Results were presented which show that the number of diversity branches $L$, 
the fading coefficient $m$, and the number of cascaded components $N$ have a significant effect on the ASEP performance. These results can easily be generalized to other diversity and modulation schemes. The expressions derived here are simple to compute and thus complete and accurate performance results can easily be obtained with negligible computational effort. In the future, we will consider the impact of correlated channels on the ASEP of the MRC M2M system.

\section{Acknowledgements}

The authors would like to thank the referees and editor for providing helpful comments and suggestions. This project was supported by the National Natural Science Foundation of China (no. 61304222 and no. 60902005), the Natural Science Foundation of Shandong Province (no. ZR2012FQ021), the Shandong Province Higher Educational Science and Technology Program (no. J12LN88), and the International Science and Technology Cooperation Program of Qingdao (no. 12-1-4-137-hz).

\section{References}

[1] S. Mumtaz, K. M. Saidul Huq, and J. Rodriguez. "Direct mobile-to-mobile communication: Paradigm for 5G," IEEE Wireless Communications, vol. 21, no. 5, (2014), pp. 14-23.

[2] L. Z. Xun, H. H. Ying, R. X. Kun, and C. W. Kui. "Influences of double-Rayleigh transmission system performance,” Journal of Xi Dian University, vol. 38, no. 5, (2011), pp.172-177.

[3] F. K. Gong, P. Ye, Y. Wang, and N. Zhang, "Cooperative mobile-to-mobile communications over double Nakagami-m fading channels," IET Communications, vol. 6, no. 18, (2012), pp. 3165-3175.

[4] G. K. Karagiannidis, N. C. Sagias, and P. T. Mathiopoulos, "N*Nakagami: A novel stochastic model for cascaded fading channels," IEEE Transactions on Communication, vol. 55, no. 8, (2007), pp. 1453-1458.

[5] H. Ilhan, I. Altunbas, and M. Uysal, "Moment generating function-based performance evaluation of amplify-and-forward relaying in $\mathrm{N} *$ Nakagami fading channels," IET Communications, vol. 5, no.1, (2011), pp. 253-263.

[6] H. C. Yang and M.S. Alouini, "MRC and GSC diversity combining with an output threshold," IEEE Transactions on Vehicular Technology, vol. 54, no.3, (2005), pp. 1081-1090.

[7] Z. X. Li, W. K. Cao, B. Liang, H.Y. Hu., "On the performance of maximal-ratio-combining over doubleRayleigh fading channels," Journal of Circuits and Systems, vol. 17, no. 3, (2012), pp. 88-93.

[8] W. Wongtraitrat and P. Supnithi, "Performance of digital modulation in double Nakagami-m fading channels with MRC diversity," IEICE Transactions on Communications, vol. E92B, no. 2, (2009), pp. 559-566.

[9] J. Malhotra, A. K. Sharma, and R. S. Kaler, "On the performance of wireless receiver in cascaded fading channel," African Journal of Information\& Communication Technology, vol. 1, no. 4, (2008), pp. 65-72.

[10] H. Than, M. Uysal, and I. Altunbas, "Cooperative diversity for intervehicular communication: Performance analysis and optimization," IEEE Transactions on Vehicular Technology, vol. 58, no. 7, (2009), pp. 3301-3310.

[11] F. K. Gong, J. Ge, and N. Zhang, "SER analysis of the mobile-relay-based M2M communication over double Nakagami-m fading channels," IEEE Communications Letters, vol. 15, no. 1, (2011),pp. 34-36.

[12] A. Annamalai, C. Tellambura, and V. K. Bhargava, "A general method for calculating error probabilities over fading channels," IEEE Transactions on Communications, vol. 53, no. 5, (2005), pp. 841-852.

[13] M. K. Simon and M. S. Alouini, Digital Communication over Fading Channels, Wiley, New York, NY. (2004).

[14] V. S. Adamchik and O. I. Marichev, "The algorithm for calculating integrals of hyper geometric type functions and its realization in reduce system," Proceedings of International Symposium on Symbolic and Algebraic Computation, (2001), pp. 212-224,Tokyo, Japan.

[15] J. G. Proakis, "Digital Communications”, McGraw-Hill, New York, NY, (2001). 


\section{Authors}
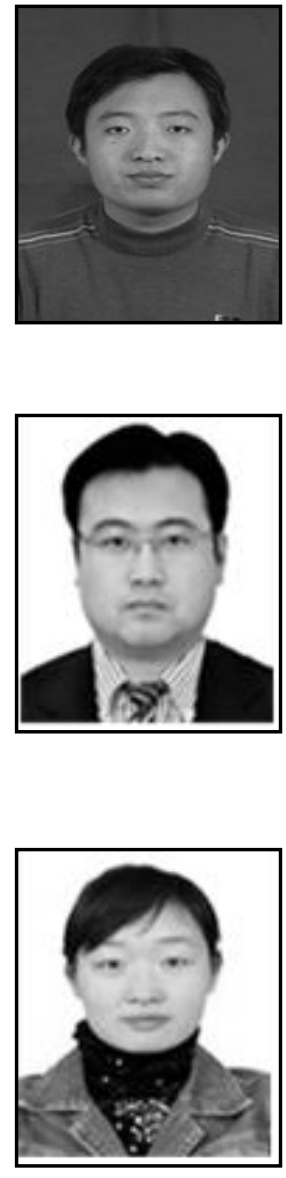

Tingting Lu was born in Qingdao, Shandong Province, China, in 1983. She received her B.S degree in Communication engineering in 2006 from HuNan University, and she received her M.S. degree in Communication and Information systems and $\mathrm{Ph} . \mathrm{D}$ in Computer application technology from Ocean University of China in 2009 and 2013 respectively. Now she is a lecturer at Ocean University of China. Her research interests are $60 \mathrm{GHz}$ wireless communication, and MIMO wireless communication

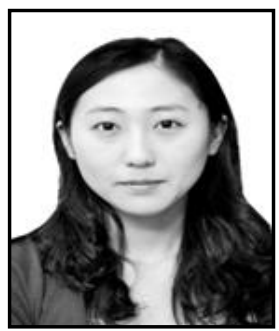

Xing Liu was born in Taian, Shandong Province, in 1990.She is currently working toward the $\mathrm{Ph}$. D. degree. Her research concerns UWB position.

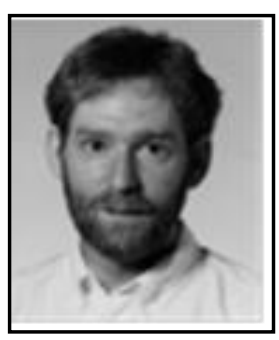

T. Aaron Gulliver received the Ph.D. degree in Electrical and Computer Engineering from the University of Victoria, Victoria, BC, and Canada in 1989. He is a professor in the Department of Electrical and Computer Engineering. In 2002 he became a Fellow of the Engineering Institute of Canada, and in 2012 a Fellow of the Canadian Academy of Engineering. He is also a senior member of IEEE. His research interests include information theory and communication theory, and ultra wideband communication. 